\title{
Endoscopic full-thickness resection of early colorectal neoplasms using an endoscopic submucosal dissection knife: a retrospective multicenter study
}

\section{(이요 $\odot$}

\author{
Authors \\ Marie-Anne Guillaumot ${ }^{1}$, Maximilien Barret ${ }^{1}$, Jérémie Jacques ${ }^{2}$, Romain Legros ${ }^{2}$, Mathieu Pioche ${ }^{3}$, Jérome Rivory ${ }^{3}$, \\ Gabriel Rahmi ${ }^{4}$, Vincent Lepilliez ${ }^{5}$, Edouard Chabrun ${ }^{6}$, Sarah Leblanc ${ }^{1}$, Stanislas Chaussade ${ }^{1}$
}

Institutions

1 Department of Gastroenterology and Digestive Oncology, Cochin Hospital, Assistance Publique Hopitaux de Paris, and University of Paris, France

2 Department of Gastroenterology, Limoges University Hospital, Limoges, France

3 Department of Gastroenterology, Edouard Herriot Hospital, Lyon, France

4 Department of Gastroenterology and Digestive Endoscopy, Georges Pompidou European Hospital, Assistance Publique - Hopitaux de Paris, and University of Paris, France

5 Department of Gastroenterology, Ramsay Générale de Santé, Hôpital Privé Jean Mermoz, Lyon, France

6 Department of Endoscopy and Gastroenterology, Bordeaux University Hospital, Bordeaux, France

submitted 21.8.2019

accepted after revision $\quad 23.1 .2020$

Bibliography

DOI https://doi.org/10.1055/a-1127-3092 |

Endoscopy International Open 2020; 08: E611-E616

(c) Georg Thieme Verlag KG Stuttgart · New York elSSN 2196-9736

Corresponding author

Maximilien Barret, Department of Gastroenterology and

Digestive Oncology, Cochin Hospital, 27 Rue du Faubourg

St Jacques, 75014 Paris, France

Fax: +33(1)58411965

maximilien.barret@aphp.fr

\section{ABSTRACT}

Background and study aims Endoscopic full-thickness resection allows resection of early gastrointestinal neoplasms not amenable to conventional endoscopic resection techniques, due to their location, presence of submucosal fibrosis, or suspected deep mural invasion. It is typically achieved using a dedicated over-the-scope device (fullthickness resection device or FTRD). The aim of our study was to evaluate the feasibility, safety, and clinical outcomes of endoscopic full-thickness resection using an endoscopic submucosal dissection (ESD) knife.

Patients and methods Consecutive patients who underwent full-thickness endoscopic resection at six tertiary care centers from August 2010 to June 2017 were retrospectively included. We conducted a comparative analysis of patient characteristics, technical success, adverse events, and time to discharge between patients treated by a full-thickness resection using an ESD knife.

Results Twenty-one procedures were performed using an ESD knife. En-bloc resection and R0 resection rates were $95.2 \%$ and $65 \%$, respectively. Clinical symptoms of perforation occurred in $66.7 \%$. There was no need for surgery or additional endoscopic procedures.

Conclusion Endoscopic full-thickness resection of early colorectal neoplasms using an ESD knife might be feasible and safe. It allows complete resection of lesions with no limitation in size. The technique may be preferable to an other-the-scope resection device in lesions larger than $20 \mathrm{~mm}$, and to surgery in selected cases of low-risk T1 colorectal carcinomas, non-lifting adenomas, submucosal tumors, or technically challenging lesion locations.

\section{Introduction}

Endoscopic submucosal dissection (ESD) and endoscopic mucosal resection (EMR) are effective techniques for removal of early neoplasia of the gastrointestinal tract by separating submucosa and muscular layer [1-4]. In some situations, such as submucosal fibrosis, previous failed resection, or submucosal invasion, these techniques may not achieve en bloc and histologically complete (R0) resection [5-8]. Endoscopic full-thickness resection could be a valid alternative to conventional surgery. The technique was inspired by the transanal endoscopic microsurgery (TEM) technique used by colorectal surgeons [9]. TEM consists of a full-thickness en bloc excision of a large rectal tumor down to the perirectal fat, with or without suture of the re- 

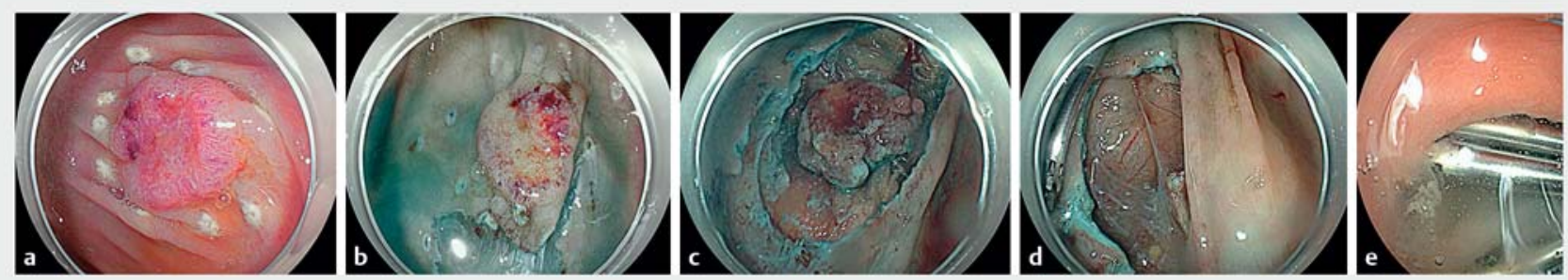

- Fig. 1 aEndoscopic full-thickness (transparietal) resection procedure. A Paris 0-Is + Ilc lesion of the lower rectum was marked with an endoscopic submucosal dissection knife and the submucosal layer was injected (Panel 1A). b Mucosal incision was achieved and c submucosal dissection performed until reaching the muscularis propria. $\mathbf{d}$ Transparietal dissection was achieved, allowing en bloc resection of a $\mathrm{T} 2$ adenocarcinoma. e The resection bed was finally closed with clips.

section bed. The main advantage of TEM is avoiding risk of impairing rectal and genitourinary function as after proctectomy and complete mesorectal excision [10-15].

A clip-assisted endoscopic full-thickness resection device (FTRD) has been shown to be feasible for such lesions in retrospective studies [16-18] and one prospective study [19], but the technique is limited by its applicability to lesions $<20 \mathrm{~mm}$ in size. Endoscopic full-thickness resection with an ESD knife performed by gastroenterologists would allow en bloc R0 resection with no limitation in terms of lesion location or size.

The aim of this retrospective multicenter study was to evaluate the feasibility, safety, and clinical outcomes of full-thickness resection with an ESD knife.

\section{Patients and methods}

\section{Indications for full-thickness endoscopic resection}

Indications for full-thickness endoscopic resection were as follows: (1) Invasive pattern on magnification chromoendoscopy and/or endoscopic ultrasound findings suggestive of deep submucosal infiltration; (2) prior endoscopic resection failure due to submucosal fibrosis or difficult lesion location; and (3) carcinoid or other subepithelial lesions.

\section{Periprocedural management}

All procedures were performed in an inpatient setting under deep sedation with propofol or general anesthesia with endotracheal intubation. Patients received a single dose of intravenous prophylactic antibiotics according to local protocols.

All resections were performed by endoscopists with extensive experience in expertise in colonoscopy, EMR and ESD ( $>30$ cases of colonic ESD).

All endoscopic procedures were performed with $\mathrm{CO}_{2}$ insufflation. Before resection, diagnostic endoscopy was performed to characterize and measure the lesion and search for an invasive pattern. Lesion margins were delineated before beginning resection using coagulation markings or indigo carmine dye spraying. After resection, the specimen was immediately collected and the resection site was assessed to check completeness of resection and search for active bleeding.
All patients were discharged minimally at postoperative day 1 and were monitored clinically for signs of bleeding or perforation.

\section{Endoscopic full-thickness resection technique}

Submucosal lifting was attempted using indigo-carminestained saline, or a lifting solution made of $5 \%$ fructose and $10 \%$ glycerol mixed with saline. Then, a circumferential mucosal incision was made. Submucosal dissection was started and converted to a full-thickness resection when impossible to complete without opening the muscularis propria ( $\mathbf{F i g . 1}$ ). Bleeding was controlled using hemostatic forceps, such as the Coagrasper (Olympus, Tokyo, Japan) in soft coagulation mode $(50 \mathrm{~W})$. Closure of the resection wound was achieved using hemoclips or an over the scope clip in the colon or proximal rectum. Desufflation of the capnoperitoneum was performed using a $14 \mathrm{G}$ needle whenever necessary.

\section{Clinicopathological characteristics}

Lesion locations were divided into six areas: rectum, sigmoid colon, descending colon, transverse colon, ascending colon, or cecum. Macroscopic tumor type was classified according to the Paris classification. The following parameters were collected: age, previous resection, tumor location, submucosal fibrosis, resection area, procedure time, and macroscopic type.

En bloc resection was defined as lesion removal in a single piece with macroscopically lesion-free lateral and vertical margins. $\mathrm{R} 0$ resection was defined as an en bloc resection with histologically complete resection, i. e. absence of neoplastic tissue at the lateral or vertical resection margins.

Clinical perforation was defined as abdominal pain, fever (temperature superior to $38.5^{\circ} \mathrm{C}$, and elevated biological inflammation markers (C-reactive protein superior to $5 \mathrm{mg} / \mathrm{L}$ and/or leucocytes superior to $10000 / \mathrm{mm}^{3}$ ) after the procedure. Computed tomography scanning was used to confirm the diagnosis and rule out other complications.

Submucosal fibrosis was defined as a whitish, dense, tissue in the submucosa without any distension or color change after submucosal injection. 


\section{Histopathologic workup evaluation}

The specimens were examined to determine histologic type, depth of invasion, lymphovascular invasion, and lateral and vertical resection margins.

Curative resection of an adenocarcinoma was defined when all of the Japanese Classification for Cancer of the Colon and Rectum [20] criteria were met: lateral and vertical resection margins free of tumor, submucosal invasion $<1,000 \mu \mathrm{m}$ from the muscularis mucosae, absence of lymphatic or vascular involvement, or poorly differentiated component. Local pathologists at each center were in charge of the pathologic assessment.

\section{Statistical analysis}

Data were summarized as median and range for discrete variables and as means and standard deviation for continuous variables, unless stated otherwise. Categorical variables are reported as percentages to describe proportions and continuous variables are reported as means. Demographics and outcomes of all patients treated by full-thickness resection with an ESD knife were recorded. Bivariate analysis was performed separately for each variable. $P$ values were calculated by the Chi-square or Fisher exact test for discrete variables, by the $t$-test or Wilcoxon test for continuous variables. All patients provided written informed consent for the procedure and the publication of the data.

\section{Results}

\section{Patients and lesions characteristics}

From August 2010 to September 2017, 21 full-thickness endoscopic resections with an ESD knife were performed in six centers. Patient characteristics are presented in $>$ Table 1.

Eight lesions were treated with Triangle Tip knife (Olympus, Tokyo, Japan), three with Hybrid-knife T-type (Erbe, Tubingen, Germany), five with Dual knife (Olympus, Tokyo, Japan), and five lesions with Jet knife (Olympus, Tokyo, Japan). Median procedure time was $126 \mathrm{~min}$ (range $=95-270$ ). Median age was 68 years (range $=38-85$ years). Two patients were on anticoagulants and three patients were on antiplatelet therapy. Lesions were located in the rectum in $76.2 \%$ of patients $(16 / 21)$, in the sigmoid colon in $14.3 \%(3 / 21)$, and in the right colon in $9.5 \%$ $(2 / 21)$. For lesions located in the rectum, they were respectively located in the distal rectum ( $<5 \mathrm{~cm}$ from the anal verge), in the mid rectum $(5-10 \mathrm{~cm})$ and in the proximal rectum $(>10 \mathrm{~cm})$ in five, three and one cases; seven cases of rectal lesions were not precisely located.

Overall, $52 \%$ of the lesions (11/21) were recurrences occurring following an EMR or an ESD of a colorectal adenoma. Lesions were categorized according to the Paris classification as type 0 -Is (2/21) or 0 -II lesions (17/21), or as subepithelial lesions (2/21). A macronodule was observed in $38 \%$ of lesions $(8 / 21)$.

Endoscopists considered pit pattern to be invasive in $43 \%$ of lesions (9/21). Mean $( \pm S D)$ size of resected lesions was $35.5 \pm$ $21 \mathrm{~mm}$ (range: $10-95 \mathrm{~mm}$ ).
- Table 1 Baseline characteristics of 21 neoplastic colorectal lesions in 21 patients treated by endoscopic full-thickness resection using an ESD knife.

\begin{tabular}{|c|c|}
\hline & $\mathbf{n}=\mathbf{2 1}$ \\
\hline Age - mean \pm SD (range), years & $65.6 \pm 10.5(38-85)$ \\
\hline Male/female ratio & $11 / 10$ \\
\hline Anticoagulant/Antithrombotic use - $n$ & $2 / 3$ \\
\hline Prior therapeutics - n (\%) & $11(52)$ \\
\hline \multicolumn{2}{|l|}{ Location - n } \\
\hline - Appendix/cecum/right/transverse/left/ & $0 / 0 / 2 / 0 / 0 / 3 / 16$ \\
\hline \multicolumn{2}{|l|}{ " sigmoid/rectum } \\
\hline - Colon vs. rectum & $5(23.8)$ vs. $16(76.2)$ \\
\hline \multicolumn{2}{|l|}{ Indication for resection - n (\%) } \\
\hline - Failed prior resection attempt & $15(71.4)$ \\
\hline - Invasive pattern & 4 (19) \\
\hline - Submucosal lesion & $2(9.5)$ \\
\hline $\begin{array}{l}\text { - Intra diverticular lesion or appendicular } \\
\text { lesion }\end{array}$ & 0 \\
\hline Size - Mean \pm SD size (range), $\mathrm{mm}$ & $46.2 \pm 2.1(10-95)$ \\
\hline \multicolumn{2}{|l|}{ Paris classification subtype $-\mathrm{n}$} \\
\hline $\begin{array}{l}\text { Is/ IIa/Is + IIa/IIa + IIb/IIa + IIc/IIb/IIc/Is + IIc/ } \\
\text { Subepithelial tumor }\end{array}$ & $3 / 3 / 5 / 0 / 3 / 0 / 3 / 3 / 1$ \\
\hline ESD, endoscopic submucosal dissection; SD, sta & rd deviation \\
\hline
\end{tabular}

\section{Technical feasibility and en bloc resection rate}

Full-thickness resection with an ESD knife was indicated based on signs of deep mural invasion in $57.1 \%(12 / 21)$, prior failed ESD in $33.3 \%(7 / 21)$ and submucosal lesions $9.5 \%(2 / 21)$. In four of seven cases of failed ESD, submucosal fibrosis was attributable to prior endoscopic resection attempts with scarring. Lesions with signs of deep submucosal infiltration were adenocarcinomas in 11 of 12 cases.

Full-thickness resection was achieved in $100 \%$ (21/21) and presence of serosa or serous or peritoneal fat was described by the pathologist in three cases. In $23.8 \%$ of cases (5/21), the resection bed was closed by conventional hemoclips (4/5) or an over-the-scope clip (Ovesco, Ovesco Endoscopy AG, Tubingen, Germany) (1/5).

En bloc resection was achieved in $90.1 \%$ (19/21), piecemeal resection in $9.5 \%$ (2/21). Plasma argon or coagulation was used as complementary treatment for macroscopically positive margins in $9.5 \%$ (2/21). Details of the endoscopic procedures are listed in $>$ Table 2.

\section{Histologic and technical outcomes}

Histologic analysis showed adenoma in $28.6 \%$ (6/21), with lowgrade dysplasia and high-grade dysplasia in one and five cases, respectively. Adenocarcinoma was reported in $61.9 \%$ (13/21), neuroendocrine tumor in $4.7 \%(1 / 21)$, and no neoplastic tissue 
- Table 2 Therapeutic outcomes after full-thickness resection of 21 early colorectal neoplasms using an ESD knife.

\begin{tabular}{|l|l|}
\hline & $\mathbf{n = 2 1}$ \\
\hline Successful resection rate $-\mathrm{n}(\%)$ & $21(100)$ \\
\hline En bloc resection rate $-\mathrm{n}(\%)$ & $19(95.2)$ \\
\hline $\mathrm{R} 0$ resection rate $-\mathrm{n}(\%)$ & $13(65)$ \\
\hline Curative resection rate for carcinoma - $\mathrm{n}(\%)$ & $2(15.4)$ \\
\hline Surgery for cancer $-\mathrm{n}(\%)$ & $5(38.4)$ \\
\hline Admission time - Mean \pm SD, (range), days & $3.1 \pm 1.95(1-8)$ \\
\hline Complications - $\mathrm{n}(\%)$ & \\
\hline - Clinical perforation & $14(66.7) 14(66.7)$ \\
\hline - Per-endoscopic desufflation/medical/ & $2 / 12 / 0$ \\
\hline surgical treatment & $0(0)$ \\
\hline - Delayed bleeding & \\
\hline ESD, endoscopic submucosal dissection & \\
\hline
\end{tabular}

was found on the specimen in $4.7 \%$ of cases $(1 / 21)$. The carcinoma was intramucosal or Tis in $9.5 \%(2 / 21))$, submucosal in $23.8 \%(5 / 21), \mathrm{T} 2$ in $23.8 \%(5 / 21)$, T3 in $4.7 \%(1 / 21)$. Histology of carcinoma was documented before the resection procedure in only 3 cases.

Resection was histologically complete (R0 resection) in $61.9 \%$ of patients (13/21). Positive deep resection margins (for adenocarcinoma) were found in two patients and positive lateral margins (with high grade dysplasia) in six. The two piecemeal resections were classified R1. Finally, among the 13 lesions containing adenocarcinoma, only two resections were considered curative according to the Japanese criteria, as shown in $>$ Table 3.

\section{Adverse events}

Clinical perforation occurred in $66.7 \%$ of cases (14/21). All patients improved with conservative treatment, including fasting and antibiotics. The need for per-procedural desufflation was not considered as a clinical perforation if no clinical consequence occurred in the following hours. No delayed bleeding requiring blood transfusion or endoscopic treatment was observed. Mean time to discharge was $3.1 \pm 2$ days. All complications were managed conservatively and there was no need for surgical intervention. No procedure-related mortality was observed.

\section{Oncologic outcomes of patients with colorectal adenocarcinomas}

In 11 of 13 resected cancers, resection was histologically incomplete and, therefore, found to be oncologically inadequate. Additional surgery or radiotherapy was recommended by the local multidisciplinary meeting. Surgical resection was actually performed in seven patients (4 with en bloc and histologically complete resections of a carcinoma with deep submucosal invasion, 2 with positive deep resection margins, 1 with positive
- Table 3 Histopathologic findings in 21 neoplastic colorectal lesions managed by full-thickness resection using an ESD knife.

\begin{tabular}{|c|c|}
\hline & $\mathrm{n}=\mathbf{2 1}$ \\
\hline Adenoma - n (\%) & $6(28.6)$ \\
\hline Cancer - n (\%) & $13(62)$ \\
\hline \multicolumn{2}{|l|}{ Tumor infiltration - $\mathrm{n}(\%)$} \\
\hline - Tis & $2(9.5)$ \\
\hline - T1 superficial ${ }^{1}$ & $1(4.8)$ \\
\hline - $\mathrm{T} 1$ deep $^{2}$ & $4(19)$ \\
\hline - $\mathrm{T2}$ & $5(23.8)$ \\
\hline - $\mathrm{T3}$ & $1(4.8)$ \\
\hline Lymphovascular involvement - n (\%) & $4(30.7)$ \\
\hline Undifferentiated adenocarcinoma - n (\%) & $1(7.6)$ \\
\hline GIST/NET - n (\%) & $1(7.6)$ \\
\hline Absence of neoplasia $-n(\%)$ & $1(7.6)$ \\
\hline \multicolumn{2}{|c|}{$\begin{array}{l}\text { ESD, endoscopic submucosal dissection; GIST/NET, gastrointestinal stroma } \\
\text { tumor/neuroendocrine tumor } \\
{ }^{1} \text { Superficial submucosal infiltration }<1000 \text { micrometers } \\
{ }^{2} \text { Deep submucosal infiltration }>1000 \text { micrometers }\end{array}$} \\
\hline
\end{tabular}

lateral margin). Overall, six of seven patients were pTON0, including the three R1 resections, after surgery. Chemoradiotherapy was performed in one patient and in another patient before surgery. Two patients did not undergo surgical resection due to heavy comorbid conditions.

\section{Discussion}

This is the first study on endoscopic full-thickness resection with an ESD knife in the colon and rectum. The study mainly included previously treated lesions in which the submucosal fibrosis caused by the initial resection attempt makes the procedure challenging with low rates of histologically complete resection achieved by endoscopy [5,21]. Endoscopic full-thickness resection could be an interesting alternative to conventional surgery.

In this retrospective multicenter study, we found that endoscopic full-thickness resection performed using an ESD knife was feasible, achieving an en-bloc resection rate of $95.2 \%$ and a $\mathrm{R} 0$ resection rate of $65 \%$, without severe complications. The clinical perforation rate of $66.7 \%$ is higher than reported after colorectal ESD or TEM. It is extremely high due to our non-restrictive definition of clinical perforation. However, each case was treated conservatively with antibiotics, pain medication and fasting, without any need for surgery or another endoscopy. Local perforation of the colorectal wall is inherent to the technique and clinical manifestations with an inflammatory response should be expected. Also, mean time to discharge of 3 days is similar to other complex endoscopic procedures: mean reported hospital stay after colorectal ESD and TEM is 3 to 5 days $[22,23]$ and 5 to 8 days, respectively $[12,24,25]$, respec- 
tively. In the future, use of novel endoscopic techniques of fullthickness suturing could further decrease the rate of complications [26, 27].

R0 resections rates of FTR using an ESD knife were slightly lower than the numbers reported using FTRD, ranging from to $79 \%$ to $87 \%[19,28,29]$. Also, FTRD induces less complications, in particular a lower rate clinical perforation, due to the immediate and safe closure of the colon. However, FTRD has two important limitations: first, the cap limits the maximum size of the lesion that can be resected and the visibility of the lateral margins during the resection, potentially increasing the risk of histologically incomplete resection [19]; second, treatment of a recurrence after use of the FTRD technique could be extremely challenging for an endoscopist because of the clip.

Of note, seven seven patients had T2 or T3 pathology on the resection specimen. This can be explained by a more difficult optical prediction of deep mural invasion in previously treated lesions. However, because three patients were unfit for surgery, endoscopic resection was the only possible curative treatment for them. Six of seven patients who underwent surgery had a tumor-free (pTONO) specimen and did not actually benefit from surgical treatment. Full-thickness endoscopic resection could indeed be preferred for patients with contraindications of surgery. The decision for surgical resection was essentially based on risk of lymphatic invasion and the necessity to perform a concomitant lymphadenectomy. Preoperative evaluation of perirectal lymph nodes by endoscopic ultrasound and magnetic resonance imaging is challenging and development of more sensitive tools to detect positive perirectal lymph nodes, such as surgical perirectal lymph node sampling [30] or sentinel lymph node biopsy, could help identify patients who will actually benefit from surgical resection [31]. Larger studies are awaited to help identify indications and determine oncologic outcomes of these procedures.

This study was limited by its retrospective nature, introducing possible selection biases, and the small number of patients. Furthermore, only expert endoscopists were involved, calling into question generalizability of the results. The relatively high rate of secondary surgical indications (47.6\%) should be put into perspective with the selection bias towards lesions difficult to treat endoscopically.

\section{Conclusion}

In conclusion, our data support that endoscopic full thickness resection in the colon and rectum using an ESD knife might be a feasible, safe, and promising resection technique. It allows complete resection of neoplastic lesions without limitation in size. The technique may be preferable to FTRD for lesions $>20$ $\mathrm{mm}$, and to surgery in selected cases of low-risk T1 colorectal carcinomas, non-lifting adenomas with failure of conventional endoscopic resection techniques, subepithelial tumors, and lesions in difficult-to-reach locations. The possible benefit of a full-thickness resection with a knife over the FTRD for large rectal lesions needs to be confirmed by further studies.

\section{Competing interests}

The authors declare that they have no conflict of interest.

\section{References}

[1] Raju GS, Lum PJ, Ross WA et al. Outcome of EMR as an alternative to surgery in patients with complex colon polyps. Gastrointest Endosc 2016; 84: 315-325

[2] Moss A, Williams S], Hourigan LF et al. Long-term adenoma recurrence following wide-field endoscopic mucosal resection (WF-EMR) for advanced colonic mucosal neoplasia is infrequent: results and risk factors in 1000 cases from the Australian Colonic EMR (ACE) study. Gut 2015; 64: 57-65

[3] Oka S, Tanaka S, Saito Y et al. Local recurrence after endoscopic resection for large colorectal neoplasia: a multicenter prospective study in Japan. Am J Gastroenterol 2015; 110: 697-707

[4] Burgess NG, Bourke MJ. Endoscopic resection of colorectal lesions: The narrowing divide between East and West. Dig Endosc Off J Jpn Gastroenterol Endosc Soc 2016; 28: 296-305

[5] Mizushima T, Kato M, Iwanaga I et al. Technical difficulty according to location, and risk factors for perforation, in endoscopic submucosal dissection of colorectal tumors. Surg Endosc 2015; 29: 133-139

[6] Hong SN, Byeon JS, Lee B-I et al. Prediction model and risk score for perforation in patients undergoing colorectal endoscopic submucosal dissection. Gastrointest Endosc 2016; 84: 98-108

[7] Xu J-F, Yang L, Jin P et al. Endoscopic approach for superficial colorectal neoplasms. Gastrointest Tumors 2016; 3: 69-80

[8] lacopini F, Saito Y, Bella A et al. Colorectal endoscopic submucosal dissection: predictors and neoplasm-related gradients of difficulty. Endosc Int Open 2017; 5: E839-E846

[9] Buess G. Review: transanal endoscopic microsurgery (TEM). J R Coll Surg Edinb 1993; 38: 239-245

[10] Casadesus D. Surgical resection of rectal adenoma: a rapid review. World J Gastroenterol 2009; 15: 3851-3854

[11] Bach SP, Hill J, Monson JRT et al. A predictive model for local recurrence after transanal endoscopic microsurgery for rectal cancer. $\mathrm{Br}$ ] Surg 2009; 96: 280-290

[12] Allaix ME, Arezzo A, Caldart M et al. Transanal endoscopic microsurgery for rectal neoplasms: experience of 300 consecutive cases. Dis Colon Rectum 2009; 52: 1831-1836

[13] Morino M, Allaix ME, Caldart M et al. Risk factors for recurrence after transanal endoscopic microsurgery for rectal malignant neoplasm. Surg Endosc 2011; 25: 3683-3690

[14] Serra-Aracil X, Mora-Lopez L, Alcantara-Moral M et al. Transanal endoscopic surgery in rectal cancer. World J Gastroenterol 2014; 20 : 11538-11545

[15] Allaix ME, Arezzo A, Morino M. Transanal endoscopic microsurgery for rectal cancer: $\mathrm{T} 1$ and beyond? An evidence-based review Surg Endosc 2016; 30: 4841-4852

[16] Schmidt A, Bauerfeind P, Gubler C et al. Endoscopic full-thickness resection in the colorectum with a novel over-the-scope device: first experience. Endoscopy 2015; 47: 719-725

[17] Schmidt A, Riecken B, Damm M et al. Endoscopic removal of over-thescope clips using a novel cutting device: a retrospective case series. Endoscopy 2014; 46: 762-766

[18] Richter-Schrag H-J, Walker C, Thimme R et al. Full thickness resection device (FTRD). Experience and outcome for benign neoplasms of the rectum and colon. Chir Z Alle Geb Oper Medizen 2016; 87: 316-325 
[19] Schmidt A, Beyna T, Schumacher B et al. Colonoscopic full-thickness resection using an over-the-scope device: a prospective multicentre study in various indications. Gut 2018; 67: 1280-1289

[20] Watanabe T, Itabashi M, Shimada Y et al. Japanese Society for Cancer of the Colon and Rectum (JSCCR) guidelines 2010 for the treatment of colorectal cancer. Int J Clin Oncol 2012; 17: 1-29

[21] Agapov M, Dvoinikova E. Factors predicting clinical outcomes of endoscopic submucosal dissection in the rectum and sigmoid colon during the learning curve. Endosc Int Open 2014; 2: E235-E240

[22] Youk E-G, Sohn DK, Hong CW et al. Early outcomes of endoscopic submucosal dissection for colorectal neoplasms according to clinical indications. Dis Colon Rectum 2016; 59: 403-410

[23] Tomiki Y, Kawai M, Takehara K et al. Clinical pathway to discharge 3 days after colorectal endoscopic submucosal dissection. Dig Endosc 2015; 27: 679-686

[24] Baatrup G, Borschitz T, Cunningham C et al. Perforation into the peritoneal cavity during transanal endoscopic microsurgery for rectal cancer is not associated with major complications or oncological compromise. Surg Endosc 2009; 23: 2680-2683

[25] Palma P, Horisberger K, Joos A et al. Local excision of early rectal cancer: is transanal endoscopic microsurgery an alternative to radical surgery? Rev Espanola Enfermedades Dig Organo Of Soc Espanola Patol Dig 2009; 101: 172-178

[26] Stavropoulos SN, Modayil R, Friedel D. Current applications of endoscopic suturing. World J Gastrointest Endosc 2015; 7: 777-789

[27] Sharaiha RZ, Kedia P, Kumta N et al. Initial experience with endoscopic sleeve gastroplasty: technical success and reproducibility in the bariatric population. Endoscopy 2015; 47: 164-166

[28] Valli PV, Mertens ], Bauerfeind P. Safe and successful resection of difficult GI lesions using a novel single-step full-thickness resection device (FTRD®). Surg Endosc 2018; 32: 289-299

[29] Van der Spek B, Haasnoot K, Meischl C et al. Endoscopic full-thickness resection in the colorectum: a single-center case series evaluating indication, efficacy and safety. Endosc Int Open 2018; 6: E1227E1234

[30] Zerz A, Müller-Stich BP, Beck J et al. Endoscopic posterior mesorectal resection after transanal local excision of T1 carcinomas of the lower third of the rectum. Dis Colon Rectum 2006; 49: 919-924

[31] Van der Pas MH, Meijer S, Hoekstra OS et al. Sentinel-lymph-node procedure in colon and rectal cancer: a systematic review and metaanalysis. Lancet Oncol 2011; 12: 540-550 